MS5-P2 Electron Nanocrystallography for Organic and Macromolecular Structure Determination

Tim Gruene ${ }^{1}$, Eric van Genderen ${ }^{1}$

1. Paul Scherrer Institute

email: tim.gruene@psi.ch

Electron crystallography has a long tradition in material science and also in 2Dcrystallography. Electrons interact much more strongly with matter and theycause about three orders of magnitude less radiation damage per diffractedquantum than X-rays. Crystals with a few to a few hundred nanometre thicknesscan be used for structure determination. These features have importantapplications for organic and (also macromolecular) structure determination: Often compounds, that represent themselves as powders to $\mathrm{X}$-ray sources, consistof single nanocrystals suitable for structure determination with electrondiffraction. In addition, crystal defects like disorder increase with crystalssize so that data from nanocrystals can produce better quality models.

We recently exploited a quantum hybrid electron detector, Timepix, to determinethe crystal structure of two organic compounds form single crystals at roomtemperature [1]. Here I will present our progress since this publication. We have improved the experimental procedure and developed methods to reducesystematic errors to such an extend that the data quality allows structuresolution with the default options of shelxt. Both structure solution andrefinement can be carried out simply with well established programs likeshelxl/shelxle. My presentation will include the effects of dynamic scatteringthat is often used as argument why electron diffraction was not practical tosolve the structures of organic or macromolecular compounds.

Currently electron nanocrystallography is at a stage comparable with X-raycrystallography 3-4 decades ago. As we exploit a vast pool of experience fromX-ray crystallography, electron nanocrystallography will soon become a new standard tool for biomolecular structure determination covering those systemsthat fail X-ray diffraction.

[1] van Genderen et al., Acta Cryst (2016), A72, 235-24

Keywords: Electron Nanocrystallography, Dynamic Scattering

\section{MS5-P3 Targeting Epigenetic \\ Vulnerabilities of Cancer Cells by Exploiting Chromatin Structure and Chemistry}

Zenita Adhireksan ${ }^{1}$, Gabriela E. Davey ${ }^{1}$, Zhujun $\mathrm{Ma}^{1}$, Giulia

Palermo $^{2}$, Tina Riedel ${ }^{2}$, Benjamin S. Murray ${ }^{3}$, Alexey A. Nazarov $^{4}$, Christian G. Hartinger ${ }^{5}$, Ursula Röthlisberger ${ }^{2}$, Paul J. Dyson $^{2}$, Curt A. Davey ${ }^{1}$

1. School of Biological Sciences, Nanyang Technological University, 60 Nanyang Drive, Singapore 637551

2. Institut des Sciences et Ingénierie Chimiques, Ecole Polytechnique Fédérale de Lausanne (EPFL), CH-1015 Lausanne, Switzerland

3. Department of Chemistry, University of Hull, Cottingham Road, Hull, HU6 7RX, United Kingdom

4. Moscow State University, Department of Chemistry, Leninskie gory, 119991 Moscow, Russia

5. School of Chemical Sciences, University of Auckland, Private Bag 92019, Auckland 1142, New Zealand

email: zenita@ntu.edu.sg

Pronounced differences in the gene expression profiles between healthy and cancerous cells coincide with extensive epigenetic distinctions in chromatin, and yet the therapeutic landscape of chromatin is largely unexplored. We thus hypothesize that compounds capable of recognizing specific features of chromatin could enable targeting weak points of cancer cells [1-3]. Recent years have witnessed a resurgence of interest in developing new metal-based anticancer agents that are more effective and have fewer side effects, with focus shifting towards metals that are alternatives to the traditional platinum. We have so far characterized the nucleosome binding activity of over 50 different non-Pt drugs and therapeutic candidates, including various $\mathrm{Ru}-, \mathrm{Os}-, \mathrm{Rh}-$ and $\mathrm{Au}$-based compounds. We observe that only one specific class of $\mathrm{Ru}$ agent preferentially forms DNA adducts, while the rest have an apparent preference for reaction at histone protein sites. In conjunction with studies on $\mathrm{Pt}$ compounds, this has revealed principles for selective targeting of different protein and DNA sites within chromatin and corresponding relationships to cytotoxicity and impact on cancer cell function. Moreover, newly designed binuclear antitumor compounds target a key regulatory site on the nucleosome, which alters chromatin structure in vitro and appears to kill cancer cells by interfering with chromatin dynamics. Beyond this, we have also discovered that two unrelated histone-targeting agents, in combination, display synergistic antitumor activity, and we can link this to a novel mechanical mechanism involving allosteric modulation in the nucleosome.

[1] C.A. Davey. 2015. Exposure to Metals Can Be Therapeutic. Chimia. 69: 125-130.

[2] B. Wu*, M.S. Ong*, M. Groessl, Z. Adhireksan, C.G. Hartinger, P.J. Dyson \& C.A. Davey. 2011. A Ruthenium Antimetastasis Agent Forms Specific Histone Protein Adducts in the Nucleosome Core. Chem. Eur. J. 17: 3562-3566.

[3] Z. Adhireksan*, G.E. Davey*, P. Campomanes*, M. Groessl, C.M. Clavel, H. Yu, A.A. Nazarov, H.F. Yeo, W.H. Ang, P. Dröge, U. Rothlisberger, P.J. Dyson \& C.A. Davey. 2014. Ligand Substitutions between Ruthenium-Cymene Compounds Can Control Protein versus DNA Targeting and Anticancer Activity. Nat. Commun. 5: 3462. 